\title{
SOME PROPERTIES OF CERTAIN CLASS OF MEROMORPHIC FUNCTIONS DEFINED BY LINEAR OPERATOR
}

\author{
P.THIRUPATHI REDDY, B. VENKATESWARLU ${ }^{\dagger}$, AND R. MAHDURI SHILPA
}

\author{
Date of Receiving : $\quad 21.09 .2019$ \\ Date of Revision : $\quad 07.04 .2020$ \\ Date of Acceptance : 10.04 .2020
}

\begin{abstract}
Making use of certain linear operator, we define a new subclass of meromorphically uniformly convex functions with positive coefficients and obtain coefficient estimates, growth and distortion theorems, extreme points, closure theorems and radius of starlikeness and convexity for the new subclass $\Sigma_{w}^{*}(\alpha, \beta, k)$.
\end{abstract}

\section{Introduction}

Let $A$ denote the class of all functions $f$ of the form

$$
f(z)=z+\sum_{n=2}^{\infty} a_{n} z^{n}
$$

in the open unit $\operatorname{disc} U=\{z \in \mathscr{C}:|z|<1\}$. Let $S$ be the subclass of $A$ consisting of univalent functions and satisfy the following usual normalization condition $f(0)=f^{\prime}(0)-1=0$. We denote by $S$ the subclass of $A$ consisting of functions $f$ which are all univalent in $U$. A function $f \in A$ is a starlike function by the order $\alpha, 0 \leq \alpha<1$, if it satisfy

$$
\operatorname{Re}\left\{\frac{z f^{\prime}(z)}{f(z)}\right\}>\alpha, z \in U .
$$

We denote this class with $S^{*}(\alpha)$.

A function $f \in A$ is a convex function by the order $\alpha, 0 \leq \alpha<1$, if it satisfy

$$
\operatorname{Re}\left\{1+\frac{z f^{\prime \prime}(z)}{f^{\prime}(z)}\right\}>\alpha, z \in U .
$$

2010 Mathematics Subject Classification. 30C45.

Key words and phrases. uniformly starlike, convex, meromorphic.

The authors express their sincere thanks to the esteemed referee(s) for their careful readings, valuable suggestions and comments, which helped them to improve the presentation of the paper.

Communicated by: Hüseyin Irmak

${ }^{\dagger}$ Corresponding author. 